\title{
Probing the Proximity of Magnetic Dichroic Signal in Electron Magnetics Circular Dichroism by Atomic Sized Electron Vortex Beam and Four Fold Astigmatic Beams.
}

\author{
Devendra Singh Negi ${ }^{1}$, Juan Carlos Idrobo ${ }^{2}$ and Jan Rusz ${ }^{3}$ \\ 1. Uppsala University, Department of Physics and Astronomy, Uppsala, Sweden \\ 2. Oak Ridge National Laboratory, Center for Nanophase Materials Sciences, Oak Ridge, Tennessee, \\ USA
}

The advent of nanotechnology, and newly emerging magnetic structures and phenomenon has pushed the functionality of electronic devices to atomic level [1,2]. Atomic level functionality further demands the tools and techniques, capable of measuring the magnetic properties at nano and atomic scale sensitivity. Several available methods, i.e, XMCD, SPSEM can sense the spatial magnetic properties, but are limited to spatial resolution of about $\sim 20 \mathrm{~nm}[3,4]$. In this context recently developed technique EMCD has shown its capability of detecting magnetic properties at nano and atomic scale [5, 6]. Due to the strong columbic interaction at atomic scale, the electron probe can cause the atomic excitation of distant atomic columns. Therefore, in order to rationalize the spatial resolution, it is necessary to study the source and nature of intrinsic delocalization [7]. We probe the proximity of magnetic signal in EMCD by electron vortex beam (EVBs) and atomic sized astigmatic beams, respectively. For present investigation antiferromagnetic $\mathrm{LaMnAsO}$ is considered as test bed material. Inelastic scattering cross section of $\mathrm{Mn}-\mathrm{L}_{3}$ edge is calculated with combined Multislice-Bloch wave approach and z-locality approximation as implemented in MATS v2 software. Convergence angle $\alpha=30 \mathrm{mrad}$, and optimized value of four fold astigmatism $\mathrm{C}_{34}(17 \mu \mathrm{m})$ is used for all calculations. Figure 1 shows the structure of LaMnAsO and a scheme of the probes used for the present study. In order to obtain maximum magnetic response from the astigmatic probe, the central intensity of astigmatic probe is restricted. Figure 2 indicates that EMCD signal intensity localizes within small scattering angle range while it smears out for higher scattering angles for astigmatic probes. For higher signal to noise ratio, EMCD, NM signal detection, EVBs is a better probe as compare to astigmatic probes (Figure 3). Higher SNR can be obtained within in small collection angle $(\sim 6-8 \mathrm{mrad})$ range. SNR can be enhance by introducing the aperture on the centre of astigmatic probe. While probing with EVBs the maximum magnetic signal arises from probed atomic column. Irrespective of material thickness, EVBs remains strongly localized on probed atomic column. Magnetic contribution arises within few nm of thickness of material. While probing with astigmatic probe majority of the magnetic signal originates from the nearest neighbour atomic column (Figure 4). Higher SNR and excellent signal localization suggests that EVBs can be an excellent probe for measuring magnetic properties at interface, crystal site specific doped magnetic impurity etc.

References:

[1] M. Fuechsle, et al. Nat. nanotechnology 7 (2012), p. 242.

[2] B. Radisavljevic, et al. Nat. nanotechnology 6 (2011), p. 147.

[3] R. Wiesendanger, et al. Science 255 (1992), p. 583.

[4] W. L. Chao et al. Nature 435 (2005), p. 1210.

[5] P. Schattschneider, et al. Nature. 441 (2006), p. 486.

[6] J. Rusz, J. C. Idrobo and S. Bhowmick, Phys. Rev. Lett. 113 (2014), p. 145501.

[7] J. Rusz et al. Phys. Rev. B 95 (2017), p. 174412. 
(a) LaMnAsO (3x3x1 Supercell ) (b)Vortex Beam (c) Astigmatic Beam
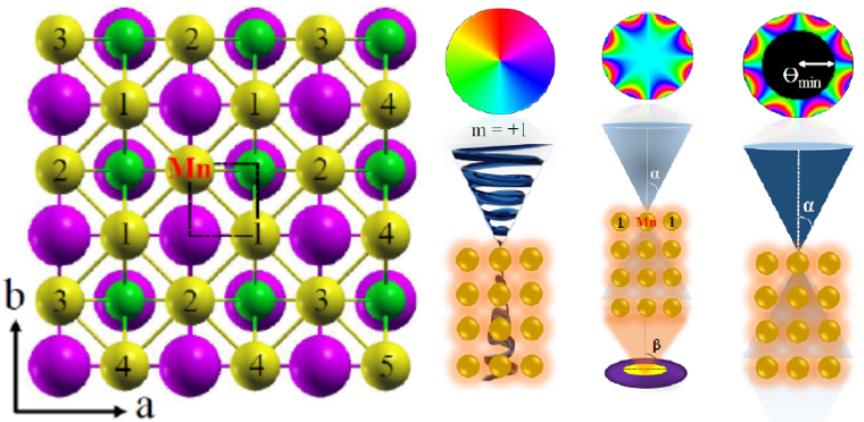

Figure 1. (a) Crystal structure of LaMnAsO and probes used for calculation.

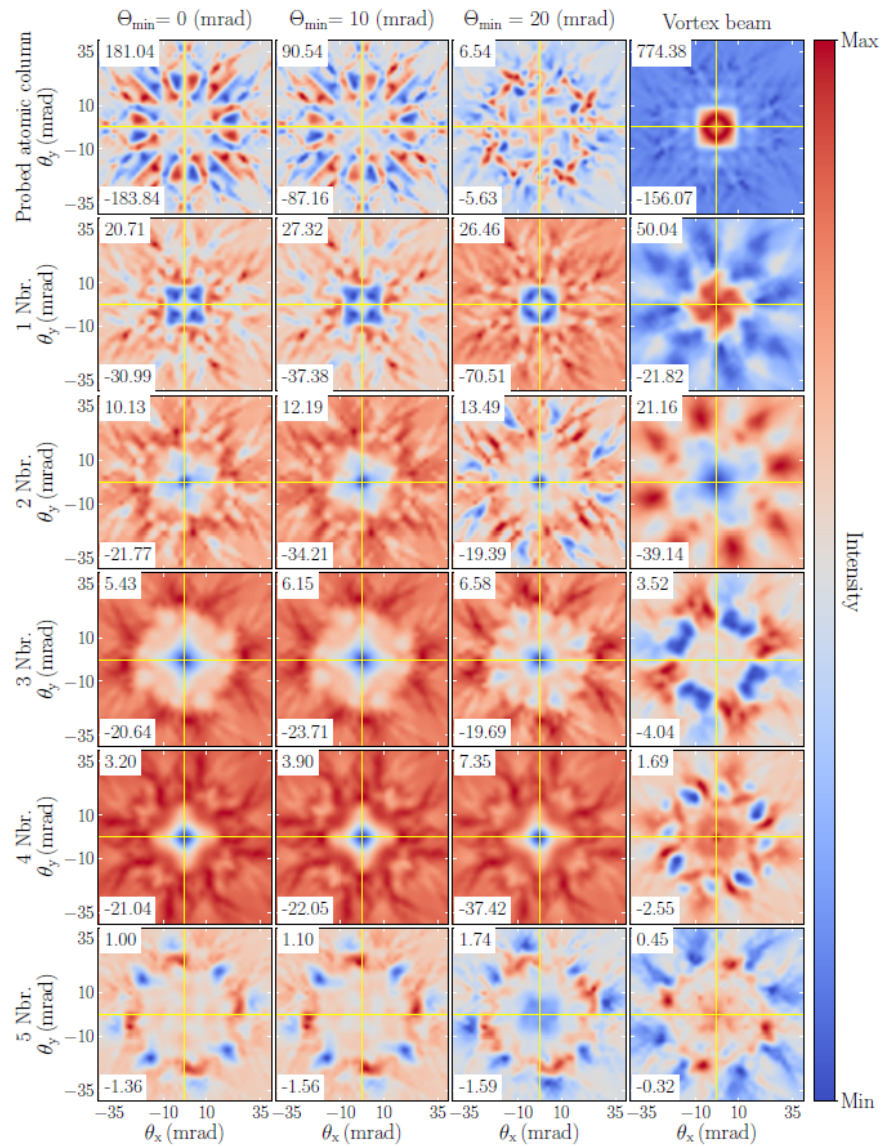

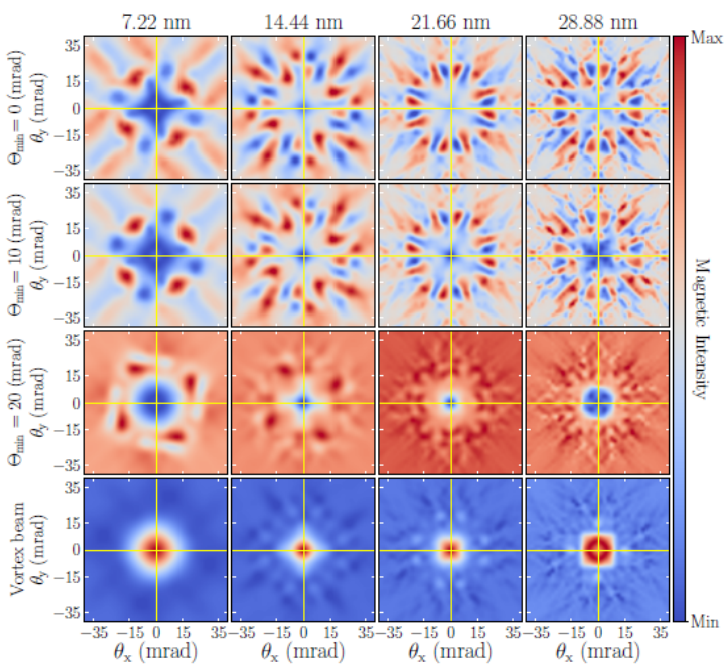

Figure 2. EMCD signal at various thickness.
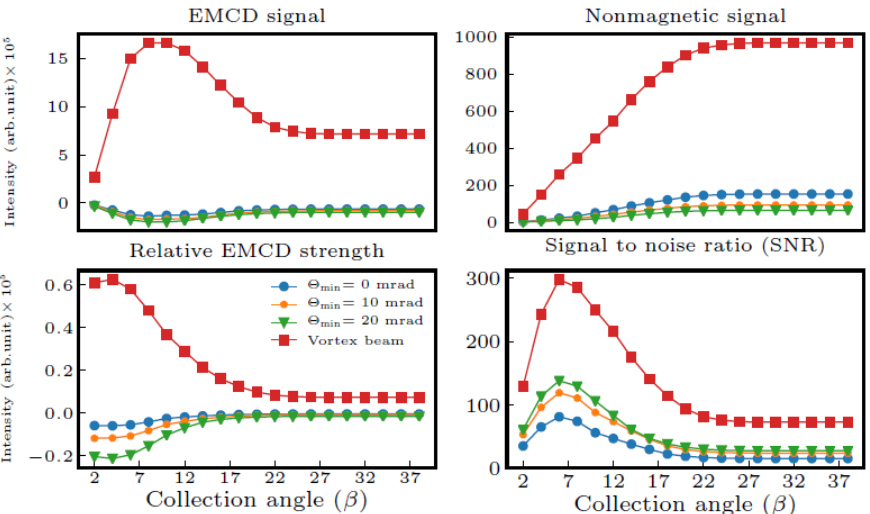

Figure 3. Radial profile of EMCD, NM, Relative EMCD \& SNR of various probes.

Figure 4. EMCD signal contribution from distant atomic column. Maximum and Minimum EMCD signal value arising from individual atomic column is placed on upper and lower section of diffraction pattern. 